\title{
ABDALlAH NABAJI \\ Construction de solutions ramifiées autour de deux hypersurfaces caractéristiques simples pour des opérateurs quasi-linéaires
}

Annales de la faculté des sciences de Toulouse $6^{e}$ série, tome $8, \mathrm{n}^{\circ} 4$ (1999), p. 629-648

<http://www.numdam.org/item?id=AFST_1999_6_8_4_629_0>

(C) Université Paul Sabatier, 1999, tous droits réservés.

L'accès aux archives de la revue «Annales de la faculté des sciences de Toulouse » (http://picard.ups-tlse.fr/ annales/) implique l'accord avec les conditions générales d'utilisation (http://www.numdam.org/conditions). Toute utilisation commerciale ou impression systématique est constitutive d'une infraction pénale. Toute copie ou impression de ce fichier doit contenir la présente mention de copyright.

\section{NumDam}

Article numérisé dans le cadre du programme Numérisation de documents anciens mathématiques http://www.numdam.org/ 


\title{
Construction de solutions ramifiées autour de deux hypersurfaces caractéristiques simples pour des opérateurs quasi-linéaires ${ }^{(*)}$
}

\author{
AbDallah NabajI ${ }^{(1)}$
}

Résumé. - Considérons dans $\mathbb{C}^{n+1}$ un opérateur différentiel quasilinéaire d'ordre deux à caractéristiques simples : $P(x, D) u=P_{p}(x, D) u+$ $f\left(x, D^{A} u\right)$ où $P_{p}$ est la partie principale de $P, A$ représente les dérivées de $u$ d'ordre un et $f$ une fonction holomorphe au voisinage de l'origine. Soit $q \in \mathbb{N}^{*}$, on suppose que : $P_{p}(x, D) u-P_{l i n}(x, D) u=O\left(u^{q}\right)$ où $P_{\text {lin }}(x, D)$ est la partie linéarisée, au voisinage de $u=0$. On étudie le problème $P(x, D) u(x)=v(x)$ où $v$ est une fonction ramifiée autour des deux hypersurfaces caractéristiques simples $K_{i}: k_{i}(x)=0,(i=1,2)$ et dont le comportement au voisinage de $K_{1} \cup K_{2}$ est de la forme : $|v(x)| \leqslant c\left|k_{1}(x)\right|^{a_{1}}\left|k_{2}(x)\right|^{a_{2}}, \quad a_{1}, a_{2} \geqslant 1 / q$. On montre alors que $u$ est ramifiée autour de $K_{1} \cup K_{2}$ et que $|u(x)| \leqslant c\left|k_{1}(x)\right|^{a_{1}+1}\left|k_{2}(x)\right|^{a_{2}+1}$.

\begin{abstract}
We consider in $\mathbb{C}^{n+1}$ a quasilinear differentiel operator of second order with simple characteristic hypersurfaces : $P(x, D) u=$ $P_{p}(x, D) u+f\left(x, D^{A} u\right)$ where $P_{p}$ is the principal part of $P, A$ represent the derivatifs of order 1 of $u$ and $f$ is a holomorphic function in a neighbor of 0 . Let $q \in \mathbb{N}^{*}$, we suppose that : $P_{p}(x, D) u-P_{l i n}(x, D) u=O\left(u^{q}\right)$ where $P_{\text {lin }}(x, D)$ is the linearzed part of $P$ near $u=0$. We study the problem $P(x, D) u(x)=v(x)$ where $v$ is a ramified function around two simple characteristic hypersurfaces, $K_{i}: k_{i}(x)=0,(i=1,2)$, and $|v(x)| \leqslant c\left|k_{1}(x)\right|^{a_{1}}\left|k_{2}(x)\right|^{a_{2}}, \quad a_{1}, a_{2} \geqslant 1 / q$. We get solutions $u$ witch are ramified around two simple characteristic hypersurfaces and $|u(x)| \leqslant$ $c\left|k_{1}(x)\right|^{a_{1}+1}\left|k_{2}(x)\right|^{a_{2}+1}$.
\end{abstract}

(*) Reçu le 27 janvier 1999, accepté le 15 septembre 1999

(1) Équipe : modélisation, E.D.P. et Analyse numérique.

F.S.T. Mohammédia B.P. 146 Mohammédia, Maroc

E-mail: nabaji@uh2m.ac.ma 


\section{A. Nabaji}

\section{Introduction}

Cet article a pour objet la construction de solution ramifiée autour de deux hypersurfaces caractéristiques simples. On supposera que ces deux hypersurfaces caractéristiques sont indépendantes du second membre.

Pour fixer les idées, considérons dans $\mathbb{C}^{n+1}$ un opérateur différentiel quasi-linéaire d'ordre deux :

$$
P(x, D) u=P_{p}(x, D) u+f\left(x, D^{A} u\right)
$$

où $P_{p}=\sum_{|\alpha|=2} a_{\alpha}\left(x, D^{A} u\right) D^{\alpha} u$ est la partie principale de $P$ et $A$ représente les dérivées de $u$ d'ordre un. On suppose que la partie principale moins la partie linéarisée, au voisinage de $u=0$, de $P$ est d'ordre $q$ où $q \in \mathbb{N}^{*}$ :

$$
P_{p}(x, D) u-P_{l i n}(x, D) u=O\left(u^{q}\right) .
$$

Supposons l'hyperplan $S: x_{0}=0$ non caractéristique et soient $K_{i}: k_{i}(x)=$ $0,(i=1,2)$ deux hypersurfaces caractéristiques simples et transverses de $P_{\text {lin }}(x, D)$, on considère alors le problème

$$
P(x, D) u=v
$$

où $v$ est une fonction dont le support singulier est $K_{1} \cup K_{2}$. On se propose de démontrer que le support singulier de $u$ est $K_{1} \cup K_{2}$. Comme dans [7], [11] et [13] des hypothèses de croissance sont nécessaires pour traiter ce problème. On supposera donc que $v$ a pour comportement au voisinage de $K_{1} \cup K_{2}$ : $|v(x)| \leqslant c\left|k_{1}(x)\right|^{a_{1}}\left|k_{2}(x)\right|^{a_{2}}$ où $a_{1}, a_{2} \geqslant 1 / q$ et on montrera alors que $u$ est ramifiée et que $|u(x)| \leqslant c\left|k_{1}(x)\right|^{a_{1}+1}\left|k_{2}(x)\right|^{a_{2}+1}$ (théorème 1.1 et 1.2).

Pour des opérateurs semi-linéaires d'ordre deux, dont la partie non linéaire est polynomiale, E. Leichtnam [9] a étudié le problème de Cauchy avec des données singulières et a construit des solutions ramifiées. Lorsque la partie non linéaire de l'opérateur semi-linéaire d'ordre deux est quelconque A. Nabaji et C. Wagschal [11] ont étudié le même problème et ont construit des solutions singulières dont le comportement est

$$
|u(x)| \leqslant c\left(\max \left(\left|k_{1}(x)\right|,\left|k_{2}(x)\right|\right)\right)^{a+2} a \geqslant 0,
$$

lorsque $|v(x)| \leqslant c\left(\max \left(\left|k_{1}(x)\right|,\left|k_{2}(x)\right|\right)\right)^{a}$. Rappelons que pour des équations linéaires d'ordre $m$, le problème de construction de solutions ramifiées d'un type plus général que celui de [9] et [11] a été étudié par E. Leichtnam [8] et récemment par $P$. Pongérard et $C$. Wagschal [14]. 
Enfin le problème de construction de solutions ramifiées autour d'une hypersurface caractéristique simple qui ne dépend pas du second membre, a été traitée, avec $q=1$, dans [4], [7], [11] et [13].

Dans [7] l'auteur a traité en plus le cas ou l'hypersurface caractéristique dépend du second membre.

La méthode d'étude du problème (2) est celle qui a été développée dans [18] , [11] et [13]; elle consiste essentiellement à réduire ce problème à un problème integro-differentiel et ceci en cherchant une solution de la forme $D_{t_{1}}^{-1} D_{t_{2}}^{-1} u\left(k_{1}(x), k_{2}(x), x\right)$ où $D_{t_{1}}^{-1}, D_{t_{2}}^{-1}$ sont deux inverses à droite des opérateurs de dérivation $D_{t_{1}}$ et $D_{t_{2}}$ (formules (2.1) et (2.2)). Il suffit alors de résoudre le problème

$$
P(x, D) D_{t_{1}}^{-1} D_{t_{2}}^{-1} u=v .
$$

Nous montrons ensuite que cette équation admet une unique solution (proposition 2.1), ceci s'obtient en montrant que l'équation (3) est équivalente à :

$$
u=\mathcal{A} u+\mathcal{F}_{2} u+\mathcal{F}_{1} u+v
$$

où $\mathcal{A}, \mathcal{F}_{2}$ et $\mathcal{F}_{1}$ résultent respectivement de $P_{\text {lin }}, P_{p}-P_{\text {lin }}$ et $f$.

Ensuite nous utilisons la fonction majorante de Lax [6] et les conditions de croissance pour construire une algèbre de Banach où l'équation (4) admet un point fixe.

\section{Résultats}

Les coordonnées d'un point $x$ de $\mathbb{C}^{n+1}$ seront notées $\left(x_{0}, x_{1}, \ldots, x_{n}\right)$.

Si $\alpha=\left(\alpha_{0}, \ldots, \alpha_{n}\right) \in \mathbb{N}^{n+1}$ est un multi-indice à composantes entières, on appelle longueur de $\alpha$ l'entier $|\alpha|=\sum_{j=0}^{n}\left|\alpha_{j}\right|$. Nous poserons $\alpha !=$ $\prod_{j=0}^{n} \alpha_{j}$ ! et pour $\xi=\left(\xi_{0}, \ldots, \xi_{n}\right) \in \mathbb{C}^{n+1} \xi^{\alpha}=\prod_{j=0}^{n} \xi_{j}^{\alpha_{j}}$. L'opérateur de dérivation par rapport à la variable $x_{j}(0 \leqslant j \leqslant n)$ sera noté $D_{j}$ et, si $\alpha \in$ $\mathbb{N}^{n+1}$ est un multi-indice de dérivation, nous poserons $D^{\alpha} u=D_{0}^{\alpha_{0}} \ldots D_{n}^{\alpha_{n}} u$. On pose

$$
A=\left\{\alpha \in \mathbb{N}^{n+1} ;|\alpha| \leqslant 1\right\}, \quad n^{\prime}=\operatorname{Card} A
$$

et

$$
D^{A} u=\left(D^{\alpha} u\right)_{\alpha \in A}, y=\left(y_{\alpha}\right)_{\alpha \in A} \in \mathbb{C}^{n^{\prime}} .
$$

On considère un opérateur différentiel quasi-linéaire du second ordre

$$
P(x, D) u=\sum_{|\alpha|=2} a_{\alpha}\left(x, D^{A} u\right) D^{\alpha} u+f\left(x, D^{A} u\right)
$$




\section{A. Nabaji}

où les fonctions $a_{\alpha}(x, y)$ et $f(x, y)$ sont holomorphes au voisinage de l'origine de $\mathbb{C}^{n+1} \times \mathbb{C}^{n^{\prime}}$.

Soit $q \in \mathbb{N}^{*}$, on supposera

$$
f(x, 0)=0 \text { pour tout } x
$$

et

$$
a_{\alpha}(x, y)-a_{\alpha}(x, 0)=\sum_{\substack{\beta \in \mathbb{N}^{n^{\prime}} \\|\beta|=q}} a_{\alpha, \beta}(x, y) y^{\beta} \text { pour tout } x
$$

où les fonctions $a_{\alpha, \beta}$ sont holomorphes au voisinage de $x=0, y=0$.

Remarque 1.1. - On remarquera que lorsque $q=1$, l'hypothèse (1.3) est vrai pour toutes les fonctions $a_{\alpha}$ holomorphes au voisinage de l'origine de $\mathbb{C}^{n+1} \times \mathbb{C}^{n^{\prime}}$. Dans [7] et [13] les auteurs ont considéré le cas où $q=1$. Notre résultat sera donc plus général.

On pose

$$
a(x, D) u=\sum_{|\alpha|=2} a_{\alpha}(x, 0) D^{\alpha} u
$$

alors

$$
P(x, D) u=a(x, D) u+\sum_{\substack {|\alpha|=2 \\
\begin{subarray}{c}{\beta \in \mathbf{N}^{n^{\prime}} \\
|\beta|=q{ | \alpha | = 2 \\
\begin{subarray} { c } { \beta \in \mathbf { N } ^ { n ^ { \prime } } \\
| \beta | = q } }\end{subarray}} a_{\alpha, \beta}\left(x, D^{A} u\right)\left(D^{A} u\right)^{\beta} D^{\alpha} u+f\left(x, D^{A} u\right) .
$$

Le symbole principal de $P(x, D)$ est alors donné par :

$$
g(x, \xi)=\sum_{|\alpha|=2} a_{\alpha}(x, 0) \xi^{\alpha} .
$$

On suppose que l'hyperplan $S: x_{0}=0$ est non caractéristique et que les caractéristiques issues de $T: x_{0}=x_{1}=0$ sont simples, c'est-à-dire que l'équation $g(0 ; \lambda, 1,0, \ldots, 0)=0$ admet deux racines $\lambda_{1}, \lambda_{2}$ distinctes. On note $k_{i}(i=1,2)$ la solution du problème de Cauchy du premier ordre

$$
\left\{\begin{array}{l}
g\left(x, \operatorname{grad} k_{i}(x)\right)=0 \\
k_{i}(x)=x_{1} \text { pour } x_{0}=0 \\
\operatorname{grad} k_{i}(0)=\left(\lambda_{i}, 1,0, \ldots, 0\right)
\end{array}\right.
$$

et $K_{i}: k_{i}(x)=0$ les hypersurfaces caractéristiques issues de $T$. 
Soit $v$ une fonction holomorphe ramifiée autour de $K_{1} \cup K_{2}$. En notant $\mathcal{R}_{\delta}$ le revêtement universel du disque pointé $\dot{D}_{\delta}=\{t \in \mathbb{C}, 0<|t|<\delta\}$ $(\delta>0)$, ceci signifie que $v$ est de la forme $v(x)=\hat{v}\left(k_{1}(x), k_{2}(x), x\right)$ où $\hat{v}: \mathcal{R}_{\delta}^{2} \times \Omega \rightarrow \mathbb{C}$ est une fonction holomorphe, $\Omega$ désignant un voisinage ouvert connexe de l'origine de $\mathbb{C}^{n+1}$. Quitte à réduire $\Omega$, on peut supposer les fonctions $k_{i}$ holomorphes dans $\Omega$ et telles que $\left|k_{i}(x)\right|<\delta$ pour $x \in \Omega$, la fonction $v$ est alors définie et holomorphe sur le revêtement universel de $\Omega-K_{1} \cup K_{2}$.

On se propose de construire des solutions de l'équation

$$
P(x, D) u=v
$$

présentant des singularités sur $K_{1} \cup K_{2}$. Pour obtenir un tel résultat, des hypothèses de croissance seront nécessaires. En plus, la présence de dérivées d'ordre deux dans la partie non linéaire de l'opérateur $P(x, D)$ nécessite, comme dans [19] et [13], une estimation de toutes les dérivées de $u\left(t_{1}, t_{2}, x\right)$ par rapport aux variables $t_{1}$ et $t_{2}$.

Soit $\pi: \mathcal{R}_{\delta} \rightarrow \dot{D}_{\delta}$ la surjection canonique. On notera $|\bullet|: \mathcal{R} \rightarrow \mathbb{R}_{+}^{*}$ la fonction $|t|=|\pi(t)|$ et on introduit la classe de fonctions suivantes :

DÉFINITION 1.1. - Soient $a_{1}, a_{2} \in \mathbb{R}$ et $\Omega$ un voisinage ouvert de l'origine de $\mathbb{C}^{n+1}$. On note $\mathcal{G}^{a_{1}, a_{2}}\left(\mathcal{R}_{\delta}^{2} \times \Omega\right)$ l'espace des fonctions $u: \mathcal{R}_{\delta}^{2} \times$ $\Omega \mapsto \mathbb{C}^{n+1}$ holomorphes telles qu'il existe une constante $c \geqslant 0$, tel que pour tout $\left(t_{1}, t_{2}, x\right) \in \mathcal{R}_{\delta}^{2} \times \Omega$ et $p_{1}, p_{2} \in \mathbb{N}$

$$
\left|D_{t_{1}}^{p_{1}} D_{t_{2}}^{p_{2}} u\left(t_{1}, t_{2}, x\right)\right| \leqslant c^{p_{1}+p_{2}+1} p_{1} ! p_{2} !\left|t_{1}\right|^{a_{1}-p_{1}}\left|t_{2}\right|^{a_{2}-p_{2}} .
$$

On a alors le théorème

THÉORÈME 1.1. - Soient $a_{1}, a_{2} \geqslant 1 / q$ et $\Omega$ un voisinage ouvert de l'origine de $\mathbb{C}^{n+1}$, il existe un voisinage ouvert $\Omega^{\prime}$ de l'origine de $\mathbb{C}^{n+1}$ tel que, pour toute fonction $v \in \mathcal{G}^{a_{1}, a_{2}}\left(\mathcal{R}_{\delta}^{2} \times \Omega\right)$, il existe $\delta_{1}>0$ et une solution $u \in \mathcal{G}^{a_{1}+1, a_{2}+1}\left(\mathcal{R}_{\delta_{1}}^{2} \times \Omega^{\prime}\right)$ du problème (1.5).

Remarque 1.2. - Si $u \in \mathcal{G}^{a_{1}, a_{2}}\left(\mathcal{R}_{\delta}^{2} \times \Omega\right)$, alors

$$
\left|u\left(t_{1}, t_{2}, x\right)\right| \leqslant c\left|t_{1}\right|^{a_{1}}\left|t_{2}\right|^{a_{2}} \text { pour tout }\left(t_{1}, t_{2}, x\right) \in \mathcal{R}_{\delta}^{2} \times \Omega \text {. }
$$

Notons $\mathcal{H}^{a_{1}, a_{2}}\left(\mathcal{R}_{\delta}^{2} \times \Omega\right)$ l'espace vectoriel de toutes les fonctions $u$ : $\mathcal{R}_{\delta}^{2} \times \Omega \mapsto \mathbb{C}$ holomorphes telles qu'il existe une constante $c \geqslant 0$,

$$
\left|u\left(t_{1}, t_{2}, x\right)\right| \leqslant c\left|t_{1}\right|^{a_{1}}\left|t_{2}\right|^{a_{2}} \text { pour tout }\left(t_{1}, t_{2}, x\right) \in \mathcal{R}_{\delta}^{2} \times \Omega .
$$




\section{A. Nabaji}

La remarque 1.1 se traduit alors $\operatorname{par} \mathcal{G}^{a_{1}, a_{2}}\left(\mathcal{R}_{\delta}^{2} \times \Omega\right) \subset \mathcal{H}^{a_{1}, a_{2}}\left(\mathcal{R}_{\delta}^{2} \times \Omega\right)$. Réciproquement, on a le lemme suivant

LEMME 1.1. - L'espace $\mathcal{H}^{a_{1}, a_{2}}\left(\mathcal{R}_{\delta}^{2} \times \Omega\right)$ est inclus dans l'espace $\mathcal{G}^{a_{1}, a_{2}}\left(\mathcal{R}_{\delta^{\prime}}^{2} \times \Omega\right)$ pour tout $0<\delta^{\prime}<\delta$.

Preuve. - Soit $0<\delta^{\prime}<\delta$, il existe $0<\lambda<1$ tel que, pour tout $t \in \mathcal{R}_{\delta^{\prime}}$, le disque centré au point $t$ et de rayon $\epsilon=\lambda|t|$ soit tracé dans $\mathcal{R}_{\delta}$. Si $\gamma_{i}$ désigne le bord du disque de centre $t_{i}$ est de rayon $\epsilon_{i}=\lambda\left|t_{i}\right|$, on a

$$
D_{t_{1}}^{p_{1}} D_{t_{2}}^{p_{2}} u\left(t_{1}, t_{2}, x\right)=\frac{-p_{1} ! p_{2} !}{4 \pi^{2}} \int_{\gamma_{1}} \int_{\gamma_{2}} \frac{u\left(\tau_{1}, \tau_{2}, x\right)}{\left(\tau_{1}-t_{1}\right)^{p_{2}+1}\left(\tau_{2}-t_{2}\right)^{p_{2}+1}} d \tau_{1} d \tau_{2}
$$

et, vu que $u \in \mathcal{H}^{a_{1}, a_{1}}\left(\mathcal{R}_{\delta}^{2} \times \Omega\right)$, il existe $c \geqslant 0$ tel que

$$
\left|D_{t_{1}}^{p_{1}} D_{t_{2}}^{p_{2}} u\left(t_{1}, t_{2}, x\right)\right| \leqslant c \frac{p_{1} ! p_{2} !}{\epsilon_{1}^{p_{1}} \epsilon_{2} p_{2}} \sup _{0 \leqslant \varphi \leqslant 2 \pi}\left|t_{1}+\epsilon_{1} e^{i \varphi}\right|^{a_{1}} \underset{0 \leqslant \varphi \leqslant 2 \pi}{\sup }\left|t_{2}+\epsilon_{2} e^{i \varphi}\right|^{a_{2}}
$$

on en déduit qu'il existe $c>0$, tel que

$$
\forall p_{1}, p_{2} \in \mathbb{N},\left|D_{t_{1}}^{p_{1}} D_{t_{2}}^{p_{2}} u\left(t_{1}, t_{2}, x\right)\right| \leqslant c^{p_{1}+p_{2}+1} p_{1} ! p_{2} !\left|t_{1}\right|^{a_{1}-p_{1}}\left|t_{2}\right|^{a_{2}-p_{2}}
$$

Du théorème 1.1 on déduit alors le

THÉORÈME 1.2. - Soient $a_{1}, a_{2} \geqslant 1 / q$ et $\Omega$ un voisinage ouvert de l'origine de $\mathbb{C}^{n+1}$, il existe un voisinage ouvert $\Omega^{\prime}$ de l'origine de $\mathbb{C}^{n+1}$ tel que, pour toute fonction $v \in \mathcal{H}^{a_{1}, a_{2}}\left(\mathcal{R}_{\delta}^{2} \times \Omega\right)$, il existe $\delta_{1}>0$ et une solution $u \in \mathcal{H}^{a_{1}+1, a_{2}+1}\left(\mathcal{R}_{\delta_{1}}^{2} \times \Omega^{\prime}\right)$ du problème (1.5).

Remarque 1.3. - Lorsque les coefficients $a_{\alpha}$ sont des fonctions holomorphes quelconque, c'est-à-dire $q=1$, les hypothèses de régularité minimales du théorème 1.1 et 1.2 sont les mêmes que celles exprimées dans [7] et [13] (pour le cas d'une ramification autour d'une hypersurface caractéristique simple qui ne dépend pas du second membre).

\section{Réduction}

Définissons d'abord des inverses à droite des opérateurs de dérivation $D_{t_{1}}$ et $D_{t_{2}}, t_{i} \in \mathcal{R}$. Si $u \in \mathcal{H}^{a_{1}, a_{2}}\left(\mathcal{R}_{\delta}^{2} \times \Omega\right)$ et $a_{i}>-1$, on pose

$$
D_{t_{1}}^{-1} u\left(t_{1}, t_{2}, x\right)=\int_{0}^{t_{1}} u\left(\tau, t_{2}, x\right) d \tau=\int_{0}^{1} u\left(s t_{1}, t_{2}, x\right) t_{1} d s
$$

et

$$
D_{t_{2}}^{-1} u\left(t_{1}, t_{2}, x\right)=\int_{0}^{t_{2}} u\left(t_{1}, \tau, x\right) d \tau=\int_{0}^{1} u\left(t_{1}, s t_{2}, x\right) t_{2} d s
$$


Remarque 2.1. - Les opérateurs $D_{t_{j}}$ et $D_{t_{i}}^{-1}, i \neq j$ commutent, ainsi que $D_{t_{1}}^{-1}$ et $D_{t_{2}}^{-1}$.

Si $u$ vérifie (1.6), on a

$$
\left|D_{t_{2}}^{-1} D_{t_{1}}^{p_{1}} u\left(t_{1}, t_{2}, x\right)\right| \leqslant \frac{c^{p_{1}+1}}{a_{2}+1} p_{1} !\left|t_{1}\right|^{a_{1}-p_{1}}\left|t_{2}\right|^{a_{2}+1}
$$

et pour $p_{2} \geqslant 1$

$$
\left|D_{t_{2}}^{p_{2}-1} D_{t_{1}}^{p_{1}} u\left(t_{1}, t_{2}, x\right)\right| \leqslant c^{p_{1}+p_{2}} p_{1} !\left(p_{2}-1\right) !\left|t_{1}\right|^{a_{1}-p_{1}}\left|t_{2}\right|^{a_{2}+1-p_{2}},
$$

ce qui prouve que la fonction $D_{t_{2}}^{-1} u$ appartient a $\mathcal{G}^{a_{1}, a_{2}+1}\left(\mathcal{R}_{\delta}^{2} \times \Omega\right)$ et de même on a $D_{t_{1}}^{-1} u \in \mathcal{G}^{a_{1}+1, a_{2}}\left(\mathcal{R}_{\delta}^{2} \times \Omega\right)$.

Nous chercherons une solution de (1.5) de la forme

$$
\mathcal{U}\left(k_{1}(x), k_{2}(x), x\right)=D_{t_{1}}^{-1} D_{t_{2}}^{-1} u\left(k_{1}(x), k_{2}(x), x\right) .
$$

Pour toute fonction holomorphe $u: \mathcal{R}_{\delta}^{2} \times \Omega \rightarrow \mathbb{C}$, on a

$$
\left\{\begin{array}{l}
a(x, D) \mathcal{U}\left(k_{1}(x), k_{2}(x), x\right)=\left[a_{0}(x)+P_{1}(x, D) D_{t_{1}}^{-1}+\right. \\
\left.+Q_{1}(x, D) D_{t_{2}}^{-1}+P_{2}(x, D) D_{t_{1}}^{-1} D_{t_{2}}^{-1}\right] u\left(t_{1}, t_{2}, x\right) \text { pour } t_{i}=k_{i}(x)
\end{array}\right.
$$

où $P_{l}$ et $Q_{l}$ sont des opérateurs différentiels linéaires d'ordre $\leqslant l$ et la fonction holomorphe $a_{0}$ est donnée par la formule

$$
a_{0}(x)=\sum_{j=0}^{n} \frac{\partial g}{\partial \xi_{j}}\left(x, D k_{1}(x)\right) \times D_{j} k_{2}(x) .
$$

Cette fonction est non nulle au voisinage de l'origine. En effet,

$$
a_{0}(0)=\frac{\partial g}{\partial \xi_{0}}\left(0, D k_{1}(0)\right) \lambda_{2}+\frac{\partial g}{\partial \xi_{1}}\left(0, D k_{1}(0)\right)
$$

et d'après l'identité d'Euler

$$
\frac{\partial g}{\partial \xi_{0}}\left(0, D k_{1}(0)\right) \lambda_{1}+\frac{\partial g}{\partial \xi_{1}}\left(0, D k_{1}(0)\right)=0
$$

d'où

$$
a_{0}(0)=\frac{\partial g}{\partial \xi_{0}}\left(0, D k_{1}(0)\right)\left(\lambda_{2}-\lambda_{1}\right)
$$

quantité non nulle vu les hypothèses. 


\section{A. Nabaji}

Quant au terme non-linéaire, on remarque que la fonction $\mathcal{U}\left(k_{1}(x)\right.$, $\left.k_{2}(x), x\right)$ et ses dérivées premières s'expriment sous forme de combinaison linéaire à coefficients fonctions holomorphes de $x$ des fonctions suivantes (calculées en $t_{i}=k_{i}(x)$ )

$$
D_{t_{i}}^{-1} u\left(t_{1}, t_{2}, x\right), D_{t_{1}}^{-1} D_{t_{2}}^{-1} u\left(t_{1}, t_{2}, x\right), D_{j} D_{t_{1}}^{-1} D_{t_{2}}^{-1} u\left(t_{1}, t_{2}, x\right) .
$$

Notons $z_{1}=\left(z_{1, j}\right)$ l'ensemble des fonctions (2.5).

Les dérivées secondes de la fonction $\mathcal{U}\left(k_{1}(x), k_{2}(x), x\right)$ s'expriment sous forme de combinaison linéaire à coefficients fonctions holomorphes de $x$ des fonctions suivantes

$$
D_{j} D_{t_{i}}^{-1} u, D_{j} D_{i} D_{t_{1}}^{-1} D_{t_{2}}^{-1} u, u, D_{t_{1}} D_{t_{2}}^{-1} u, D_{t_{2}} D_{t_{1}}^{-1} u .
$$

Nous notons $z_{2}=\left(z_{2, \kappa}\right)$ l'ensemble des fonctions (2.6).

On a alors

$$
\sum_{|\alpha|=2} \sum_{\substack{\beta \in \mathbb{N}^{n^{\prime}} \\|\beta|=q}} a_{\alpha, \beta}\left(x, D^{A} \mathcal{U}\right)\left(D^{A} \mathcal{U}\right)^{\beta} D^{\alpha} \mathcal{U}=\sum_{\substack{\kappa \in \mathbb{N}^{n+4},|\beta|=q \\ \beta, \beta}} h_{\kappa, \beta}\left(x, z_{1}\right) z_{1}^{\beta} z_{2, \kappa}
$$

où les fonctions $h_{\kappa, \beta}$ sont holomorphes au voisinage de $x=0, z_{1}=0$. Nous noterons $\mathcal{F}_{i}: u \mapsto \mathcal{F}_{i} u$ les applications

$$
\left(\mathcal{F}_{1} u\right)\left(t_{1}, t_{2}, x\right)=f\left(x, z_{1}\right)
$$

et

$$
\left(\mathcal{F}_{2} u\right)\left(t_{1}, t_{2}, x\right)=\sum_{\substack{\kappa \\|\beta|=q}} h_{\kappa, \beta}\left(x, z_{1}\right) z_{1}^{\beta} z_{2, \kappa}
$$

Le problème (1.5) s'écrit ( modulo un changement de notation)

$$
u\left(t_{1}, t_{2}, x\right)=(\mathcal{A} u)\left(t_{1}, t_{2}, x\right)+\left(\mathcal{F}_{1} u\right)\left(t_{1}, t_{2}, x\right)+\left(\mathcal{F}_{2} u\right)\left(t_{1}, t_{2}, x\right)+v\left(t_{1}, t_{2}, x\right)
$$

où $\mathcal{A}$ est l'application linéaire

$$
\begin{aligned}
(\mathcal{A} u)\left(t_{1}, t_{2}, x\right)=\left(P_{1}(x, D) D_{t_{1}}^{-1}+Q_{1}(x, D)\right. & D_{t_{2}}^{-1} \\
& \left.+P_{2}(x, D) D_{t_{1}}^{-1} D_{t_{2}}^{-1}\right) u\left(t_{1}, t_{2}, x\right) .
\end{aligned}
$$

Nous démontrons alors la

Proposition 2.1. - Soient $a_{1}, a_{2} \geqslant 1 / q$ et $\Omega$ un voisinage ouvert de l'origine de $\mathbb{C}^{n+1}$, il existe un voisinage ouvert $\Omega^{\prime}$ de l'origine de $\mathbb{C}^{n+1}$ tel que, pour toute fonction $v \in \mathcal{G}^{a_{1}, a_{2}}\left(\mathcal{R}_{\delta}^{2} \times \Omega\right)$, il existe $\delta_{1}>0$ et une unique solution $u \in \mathcal{G}^{a_{1}, a_{2}}\left(\mathcal{R}_{\delta_{1}}^{2} \times \Omega^{\prime}\right)$ du problème (2.9). 


\section{Algèbres de Banach}

La démonstration de la proposition 2.1 repose sur le théorème du point fixe dans des espaces de Banach définis de la façon suivante. On utilise la fonction majorante de Lax [6] déjà utilisée dans [11], [13] et [18], on pose $\theta(\xi)=\sum_{n=0}^{\infty} \frac{\xi^{n}}{(n+1)^{2}}$ et pour $R>0, \varphi_{R}(\xi)=\theta(\xi / R)$. Nous utiliserons la proposition 2.1 et les lemmes 2.4 et 9.2 de [18].

Proposition 3.1. - Il existe une constante $c_{0} \geqslant 0$ telle que

$$
\varphi_{R}^{2}(\xi) \ll c_{0} \varphi_{R}(\xi)
$$

LEMME 3.2. - Soit $\eta>1$, il existe une constante $c=c(\eta) \geqslant 0$ telle que

$$
\frac{\eta R}{\eta R-\xi} \ll c \varphi_{R}(\xi)
$$

Si $a$ est une fonction holomorphe et bornée dans le polydisque

$$
\Delta_{\eta R}=\left\{x \in \mathbb{C}^{n+1} ; \max _{0 \leqslant j \leqslant n}\left|x_{j}\right|<\eta R\right\},
$$

on a d'après les inégalités de Cauchy

$$
a \ll M \frac{\eta R}{\eta R-\xi} \ll M c(\eta) \varphi_{R}(\xi)
$$

où $\xi=x_{0}+x_{1}+\cdots+x_{n}$ et $M=\sup _{x \in \Delta_{\eta R}}|a(x)|$.

LEMME 3.3. - Soit $\eta>1$, il existe une constante $c=c(\eta) \geqslant 0$ telle que

$$
\frac{\eta R}{\eta R-\xi} \ll c \eta^{p} \frac{R^{p}}{p !} D^{p} \varphi_{R}(\xi) \text { pour tout } p \geqslant 0 \text {. }
$$

Le lemme 4.6 de [11] peut s'écrire ainsi

LEMME 3.4. - Il existe une constante $c \geqslant 0$ telle que

$$
D^{p} \varphi_{R}(\xi) \ll c R D^{p+1} \varphi_{R}(\xi) \text { pour tout } p \geqslant 0 .
$$

Considérons une fonction $u \in \mathcal{G}^{a_{1}, a_{2}}\left(\mathcal{R}_{\delta}^{2} \times \Omega\right)$ et soit $\eta>1, R>0$ tel que $\Omega$ contienne le polydisque $\Delta_{\eta R}$. D'après les inégalités de Cauchy, si $u$ vérifie (1.6) on a pour tout $t_{1}, t_{2} \in \mathcal{R}_{\delta}$ et $p_{1}, p_{2} \in \mathbb{N}$,

$$
D_{t_{1}}^{p_{1}} D_{t_{2}}^{p_{2}} u\left(t_{1}, t_{2}, x\right) \ll c^{p_{1}+p_{2}+1} p_{1} ! p_{2} !\left|t_{1}\right|^{a_{1}-p_{1}}\left|t_{2}\right|^{a_{2}-p_{2}} \frac{\eta R}{\eta R-\xi},
$$




\section{A. Nabaji}

et d'après le lemme 3.3 , il existe donc une constante $c \geqslant 0$ telle que

$$
D_{t_{1}}^{p_{1}} D_{t_{2}}^{p_{2}} u\left(t_{1}, t_{2}, x\right) \ll c^{p_{1}+p_{2}+1}\left|t_{1}\right|^{a_{1}-p_{1}}\left|t_{2}\right|^{a_{2}-p_{2}} D^{p_{1}+p_{2}} \varphi_{R}(\xi) .
$$

Réciproquement, soit $u$ une fonction holomorphe au voisinage de $\mathcal{R}_{\delta}^{2} \times$ $\{0\}$ vérifiant (3.4); la fonction $u$ est holomorphe dans $\mathcal{R}_{\delta}^{2} \times \Omega_{R}$ où $\Omega_{R}=$ $\left\{x \in \mathbb{C}^{n+1} ;\left|x_{0}\right|+\left|x_{1}\right|+\cdots+\left|x_{n}\right|<R\right\}$ et, pour tout $\left(t_{1}, t_{2}, x\right) \in \mathcal{R}_{\delta}^{2} \times \Omega_{r}$, $0<r<R$,

$$
\left|D_{t_{1}}^{p_{1}} D_{t_{2}}^{p_{2}} u\left(t_{1}, t_{2}, x\right)\right| \leqslant c^{p_{1}+p_{2}+1}\left|t_{1}\right|^{a_{1}-p_{1}}\left|t_{2}\right|^{a_{2}-p_{2}} D^{p_{1}+p_{2}} \varphi_{R}(r),
$$

où $D^{p_{1}+p_{2}} \varphi_{R}(r) \leqslant c^{p_{1}+p_{2}+1}\left(p_{1}+p_{2}\right) ! \leqslant(2 c)^{p_{1}+p_{2}+1} p_{1}$ ! $p_{2}$ !, ce qui prouve que $u$ appartient à l'espace $\mathcal{G}^{a_{1}, a_{2}}\left(\mathcal{R}_{\delta}^{2} \times \Omega_{r}\right)$. Ceci conduit à la définition suivante.

DÉfinition 3.1. - Soient $\delta>0, a_{1}, a_{2} \in \mathbb{R}$ et $R, L, \omega>0$ tels que $2 \omega \delta<R$. On note $\mathcal{G}^{a_{1}, a_{2}}(\delta, L, \omega)$ l'ensemble des fonctions $u$ holomorphes au voisinage de $\mathcal{R}_{\delta}^{2} \times\{0\}$ telles qu'il existe une constante $c \geqslant 0$ telle que, pour tout $t_{1}, t_{2} \in \mathcal{R}_{\delta}$ et $p_{1}, p_{2} \in \mathbb{N}$, on a

$$
D_{t_{1}}^{p_{1}} D_{t_{2}}^{p_{2}} u\left(t_{1}, t_{2}, x\right) \ll c L^{p_{1}+p_{2}}\left|t_{1}\right|^{a_{1}-p_{1}}\left|t_{2}\right|^{a_{2}-p_{2}} D^{p_{1}+p_{2}} \varphi_{R}\left(\omega\left(\left|t_{1}\right|+\left|t_{2}\right|\right)+\xi\right) .
$$

Il est clair que $\mathcal{G}^{a_{1}, a_{2}}(\delta, L, \omega)$ est un espace vectoriel et que la plus petite constante $c$ pour laquelle (3.5) a lieu est une norme sur cet espace vectoriel.

Note. - Nous noterons indifféremment $c$ toute constante qui ne dépend pas des paramètres $\delta, \omega$ et $L$.

Grâce à un raisonnement analogue à celui fait pour le lemme 4.2 de [11], on vérifie que toute fonction de l'espace $\mathcal{G}^{a_{1}, a_{2}}(\delta, L, \omega)$ est holomorphe sur l'ouvert

$$
\mathcal{U}=\left\{\left(t_{1}, t_{2}, x\right) \in \mathcal{R}_{\delta}^{2} \times \mathbb{C}^{n+1} ; \omega\left(\left|t_{1}\right|+\left|t_{2}\right|\right)+\left|x_{0}\right|+\left|x_{1}\right|+\cdots+\left|x_{n}\right|<R\right\} .
$$

Remarque 3.1. - Grâce à un raisonnement analogue à celui fait pour la proposition 4.3 de [11], on vérifie que $\mathcal{G}^{a_{1}, a_{2}}(\delta, L, \omega)$ est un espace de Banach.

Remarque 3.2. - Si $a_{1}, a_{2}, b_{1}, b_{2} \in \mathbb{R}$ et $a_{i} \leqslant b_{i}$, alors $\mathcal{G}^{b_{1}, b_{2}}(\delta, L, \omega) \subset$ $\mathcal{G}^{a_{1}, a_{2}}(\delta, L, \omega)$ et l'injection canonique est linéaire continue de norme $\leqslant$ $\delta^{b_{1}+b_{2}-a_{1}-a_{2}}$. 
Proposition 3.5. - Soient $a_{1}, a_{2}, b_{1}, b_{2} \in \mathbb{R}$ et $(u, v) \in\left(\mathcal{G}^{a_{1}, a_{2}} \times \mathcal{G}^{b_{1}, b_{2}}\right)$ $(\delta, L, \omega)$, alors $u v \in \mathcal{G}^{a_{1}+b_{1}, a_{2}+b_{2}}(\delta, L, \omega)$ et

$$
\|u v\|_{\mathcal{G}^{a_{1}+b_{1}, a_{2}+b_{2}}} \leqslant c_{0}\|u\|_{\mathcal{G}^{a_{1}, a_{2}}}\|v\|_{\mathcal{G}^{b_{1}, b_{2}}}
$$

où c $c_{0}$ est la constante telle que $\varphi_{R}^{2} \ll c_{0} \varphi_{R}$.

Preuve. - Pour tout $p_{1}, p_{2} \in \mathbb{N}$, on a

$$
D_{t_{1}}^{p_{1}} D_{t_{2}}^{p_{2}}(u v)=\sum_{i=0}^{p_{1}} \sum_{j=0}^{p_{2}}\left(\begin{array}{c}
p_{1} \\
i
\end{array}\right)\left(\begin{array}{c}
p_{2} \\
j
\end{array}\right) D_{t_{1}}^{p_{1}-i} D_{t_{2}}^{p_{2}-j} u D_{t_{1}}^{i} D_{t_{2}}^{j} v
$$

d'où

$$
\begin{gathered}
D_{t_{1}}^{p_{1}} D_{t_{2}}^{p_{2}}(u v) \ll\|u\|\|v\| L^{p_{1}+p_{2}}\left|t_{1}\right|^{a_{1}+b_{1}-p_{1}}\left|t_{2}\right|^{a_{2}+b_{2}-p_{2}} \\
\sum_{i=0}^{p_{1}} \sum_{j=0}^{p_{2}}\left(\begin{array}{c}
p_{1} \\
i
\end{array}\right)\left(\begin{array}{c}
p_{2} \\
j
\end{array}\right)\left(D^{p_{1}+p_{2}-i-j} \varphi_{R} D^{i+j} \varphi_{R}\right)\left(\omega\left(\left|t_{1}\right|+\left|t_{2}\right|\right)+\xi\right)
\end{gathered}
$$

et, d'après $(3.1)$, on a

$$
\begin{gathered}
\sum_{i=0}^{p_{1}} \sum_{j=0}^{p_{2}}\left(\begin{array}{c}
p_{1} \\
i
\end{array}\right)\left(\begin{array}{c}
p_{2} \\
j
\end{array}\right)\left(D^{p_{1}+p_{2}-i-j} \varphi_{R} D^{i+j} \varphi_{R}\right)\left(\omega\left(\left|t_{1}\right|+\left|t_{2}\right|\right)+\xi\right) \ll \\
c_{0} D^{p_{1}+p_{2}} \varphi_{R}\left(\omega\left(\left|t_{1}\right|+\left|t_{2}\right|\right)+\xi\right),
\end{gathered}
$$

ce qui permet de conclure.

Q.E.D.

Si $u, v \in \mathcal{G}^{a_{1}, a_{2}}(\delta, L, \omega)$ alors $u v \in \mathcal{G}^{2 a_{1}, 2 a_{2}}(\delta, L, \omega) \subset \mathcal{G}^{a_{1}, a_{2}}(\delta, L, \omega)$ lorsque $a_{1}, a_{2} \geqslant 0$. On en déduit que $\mathcal{G}^{a_{1}, a_{2}}(\delta, L, \omega)$ est une algèbre de Banach.

Nous aurons besoin du lemme suivant

LEMME 3.6. - Soient $F(x, u)$ une fonction holomorphe et bornée par $M$ sur un polydisque centré en $0 \in \mathbb{C}^{n+1} \times \mathbb{C}^{q}$ de rayon $\eta R, R>0$, $\eta>1$ et $u_{1}, \ldots, u_{q} \in \mathcal{G}^{0,0}(\delta, L, \omega)$ telles que $c_{0}\left\|u_{i}\right\|_{\mathcal{G}^{0,0}}<R$, alors $v=$ $F\left(x, u_{1}, \ldots, u_{q}\right) \in \mathcal{G}^{0,0}(\delta, L, \omega)$ et

$$
\|v\| \leqslant c M \prod_{i=1}^{q} \frac{R}{R-c_{0}\left\|u_{i}\right\|}
$$

où la constante $c$ ne dépend que de $\eta$. 
Preuve. - Pour tout $t_{1}, t_{2} \in \mathcal{R}_{\delta}$, on a

$$
u_{i} \ll\left\|u_{i}\right\| \varphi_{R}\left(\omega\left(\left|t_{1}\right|+\left|t_{2}\right|\right)+\xi\right)
$$

d'où

$$
\left|u_{i}(t, 0)\right| \leqslant\left\|u_{i}|| \varphi_{R}\left(\omega\left(\left|t_{1}\right|+\left|t_{2}\right|\right)\right) \leqslant\right\| u_{i} \| \varphi_{R}(R) ;
$$

de la relation $\varphi_{R}^{2} \ll c_{0} \varphi_{R}$, on déduit $\varphi_{R}(R) \leqslant c_{0}$, d'où $\left|u_{i}(t, 0)\right| \leqslant c_{0}\left\|u_{i}\right\|<$ $R$. Ceci prouve que la fonction $v=F\left(x, u_{1}, \ldots, u_{q}\right)$ est bien définie et holomorphe au voisinage de $\mathcal{R}_{\delta}^{2} \times\{0\}$ et on a

$$
v=F\left(x, u_{1}, \ldots, u_{q}\right)=\sum_{\alpha \in \mathbb{N}^{q}} F_{\alpha}(x) u_{1}^{\alpha_{1}} \ldots u_{q}^{\alpha_{q}},
$$

où les fonctions $F_{\alpha}$ sont holomorphes, bornées par $M R^{-|\alpha|}$ sur le polydisque $\Delta_{\eta R}$ et donc appartiennent à l'espace $\mathcal{G}^{0,0}(\delta, L, \omega)$ et de norme $\leqslant c M R^{-|\alpha|}$ où $c=c(\eta)$ d'après le lemme 3.2. Lorsque $\alpha \neq 0$, on a d'après la proposition $3.5 u^{\alpha} \in \mathcal{G}^{0,0}(\delta, L, \omega)$ et $\left\|u^{\alpha}\right\| \leqslant c_{0}^{|\alpha|-1} \prod_{i=1}^{q}\left\|u_{i}\right\|^{\alpha_{i}}$, d'où $F_{\alpha} u^{\alpha} \in$ $\mathcal{G}^{0,0}(\delta, L, \omega)$ et

$$
\left\|F_{\alpha} u^{\alpha}\right\| \leqslant c M \prod_{i=1}^{q}\left(\frac{c_{0}\left\|u_{i}\right\|}{R}\right)^{\alpha_{i}} .
$$

Cette inégalité vaut encore pour $\alpha=0$. Ceci montre que la famille $\left(F_{\alpha} u^{\alpha}\right)$ est absolument sommable dans l'espace $\mathcal{G}^{0,0}(\delta, L, \omega)$ et par conséquent

$$
\|v\| \leqslant c M \prod_{i=1}^{q} \frac{R}{R-c_{0}\left\|u_{i}\right\|}
$$

Q.E.D.

Nous imposerons d'abord à $R>0$ de vérifier la propriété suivante : tous les coefficients des opérateurs apparaissant dans $\mathcal{A}$ sont holomorphes et bornés sur le polydisque $\Delta_{\eta R}$ où $\eta>1$.

Proposition 3.7. - Soient $a_{1}, a_{2}>-1, \omega \geqslant 1, L \geqslant 1$ et $0<\delta \leqslant 1$ alors l'application $\mathcal{A}$ induit un endomorphisme de l'espace $\mathcal{G}^{a_{1}, a_{2}}(\delta, L, \omega)$ dont la norme est $\leqslant c \max \left(\omega^{-1}, L^{-1}\right)$ et la constante $c$ ne dépend que de $\eta, R$ et de la borne supérieure sur $\Delta_{\eta}$ des coefficients des opérateurs $P_{1}$, $Q_{1}$ et $P_{2}$, figurant dans $\mathcal{A}$.

Preuve. - Il s'agit de vérifier la proposition pour des opérateurs de la forme $a(x) D^{\alpha} D_{t_{i}}^{-1}$ et $a(x) D^{\beta} D_{t_{1}}^{-1} D_{t_{2}}^{-1}$ où $|\alpha| \leqslant 1,|\beta| \leqslant 2$ et $a(x)$ est une fonction holomorphe et bornée sur $\Delta_{\eta R}$. Soit $u \in \mathcal{G}^{a_{1}, a_{2}}(\delta, L, \omega)$, on a $D_{t_{1}}^{p_{1}} D_{t_{2}}^{p_{2}} D^{\alpha} D_{t_{1}}^{-1} u=D^{\alpha} D_{t_{1}}^{p_{1}-1} D_{t_{2}}^{p_{2}} u$. 
Si $p_{1} \geqslant 1$, on a

$D_{t_{1}}^{p_{1}-1} D_{t_{2}}^{p_{2}} u$

$\ll\|u\| L^{p_{1}+p_{2}-1}\left|t_{1}\right|^{a_{1}-p_{1}+1}\left|t_{2}\right|^{a_{2}-p_{2}} D^{p_{1}+p_{2}-1} \varphi_{R}\left(\omega\left(\left|t_{1}\right|+\left|t_{2}\right|\right)+\xi\right)$.

En dérivant, on obtient

$D^{\alpha} D_{t_{1}}^{p_{1}-1} D_{t_{2}}^{p_{2}} u$

$\ll c\|u\| L^{p_{1}+p_{2}-1}\left|t_{1}\right|^{a_{1}-p_{1}+1}\left|t_{2}\right|^{a_{2}-p_{2}} D^{p_{1}+p_{2}} \varphi_{R}\left(\omega\left(\left|t_{1}\right|+\left|t_{2}\right|\right)+\xi\right)$

en effet, si $|\alpha|=1$ ceci est vrai avec $c=1$ et si $|\alpha|=0$ on a, d'après (3.3) $D^{p_{1}+p_{2}-1} \varphi_{R} \ll c D^{p_{1}+p_{2}} \varphi_{R}$. Or $\left|t_{1}\right|<\delta \leqslant 1$, d'où $D_{t_{1}}^{p_{1}} D_{t_{2}}^{p_{2}} D^{\alpha} D_{t_{1}}^{-1} u$

$\ll c L^{-1}|| u \| L^{p_{1}+p_{2}}\left|t_{1}\right|^{a_{1}-p_{1}}\left|t_{2}\right|^{a_{2}-p_{2}} D^{p_{1}+p_{2}} \varphi_{R}\left(\omega\left(\left|t_{1}\right|+\left|t_{2}\right|\right)+\xi\right)$.

Si $p_{1}=0$, on a

$$
D_{t_{2}}^{p_{2}} u \ll\|u\| L^{p_{2}}\left|t_{2}\right|^{a_{2}-p_{2}} \sum_{j=0}^{\infty} \frac{\omega^{j}\left|t_{1}\right|^{a_{1}+j}}{j !} D^{p_{2}+j} \varphi_{R}\left(\omega\left|t_{2}\right|+\xi\right)
$$

d'où

$D_{t_{1}}^{-1} D_{t_{2}}^{p_{2}} u$

$$
\ll\|u\| L^{p_{2}}\left|t_{2}\right|^{a_{2}-p_{2}} \sum_{j=0}^{\infty} \frac{j+1}{a_{1}+j+1} \frac{\omega^{j}\left|t_{1}\right|^{a_{1}+j+1}}{(j+1) !} D^{p_{2}+j} \varphi_{R}\left(\omega\left|t_{2}\right|+\xi\right)
$$

et $s u p_{j \geqslant 0} \frac{j+1}{a_{1}+j+1}$ étant fini, on en déduit que

$$
D_{t_{1}}^{-1} D_{t_{2}}^{p_{2}} u \ll c\|u\| L^{p_{2}}\left|t_{1}\right|^{a_{1}}\left|t_{2}\right|^{a_{2}-p_{2}} \sum_{j=0}^{\infty} \frac{\omega^{j}\left|t_{1}\right|^{j+1}}{(j+1) !} D^{p_{2}+j} \varphi_{R}\left(\omega\left|t_{2}\right|+\xi\right) .
$$

En dérivant, en obtient

$$
\begin{aligned}
D^{\alpha} D_{t_{1}}^{-1} D_{t_{2}}^{p_{2}} u & \\
& \ll c\|u\| L^{p_{2}}\left|t_{1}\right|^{a_{1}}\left|t_{2}\right|^{a_{2}-p_{2}} \sum_{j=0}^{\infty} \frac{\omega^{j}\left|t_{1}\right|^{j+1}}{(j+1) !} D^{p_{2}+j+|\alpha|} \varphi_{R}\left(\omega\left|t_{2}\right|+\xi\right)
\end{aligned}
$$

il existe, donc une constante $c \geqslant 0$ telle que

$$
\begin{aligned}
& D_{t_{2}}^{p_{2}} D^{\alpha} D_{t_{1}}^{-1} u \ll\|u\| L^{p_{2}}\left|t_{1}\right|^{a_{1}}\left|t_{2}\right|^{a_{2}-p_{2}} \sum_{j=0}^{\infty} \frac{\omega^{j}\left|t_{1}\right|^{j+1}}{(j+1) !} D^{p_{2}+j+1} \varphi_{R}\left(\omega\left|t_{2}\right|+\xi\right) \\
& \ll \frac{c}{\omega}\|u\| L^{p_{2}}\left|t_{1}\right|^{a_{1}}\left|t_{2}\right|^{a_{2}-p_{2}} D^{p_{2}} \varphi_{R}\left(\omega\left(\left|t_{1}\right|+\left|t_{2}\right|\right)+\xi\right) . \\
&-641-
\end{aligned}
$$




\section{A. Nabaji}

Ceci prouve que $D^{\alpha} D_{t_{1}}^{-1} u$ appartient à l'espace $\mathcal{G}^{a_{1}, a_{2}}(\delta, L, \omega)$ et que sa norme est $\leqslant c \max \left(L^{-1}, \omega^{-1}\right)\|u\|$. Quant à la fonction $a$, elle appartient à l'espace $\mathcal{G}^{0,0}(\delta, L, \omega)$ et la proposition 3.5 permet de conclure.

On a évidemment le même résultat pour l'opérateur $a(x) D^{\alpha} D_{t_{2}}^{-1}$.

Soit $\beta \in \mathbb{N}$ tel que $|\beta| \leqslant 2$, on peut écrire $\beta=\alpha+\alpha^{\prime}$ tel que $|\alpha|,\left|\alpha^{\prime}\right| \leqslant 1$ et

$$
D^{\beta} D_{t_{1}}^{-1} D_{t_{2}}^{-1} u=D^{\alpha^{\prime}} D_{t_{2}}^{-1} D^{\alpha} D_{t_{1}}^{-1} u .
$$

Donc $a(x) D^{\beta} D_{t_{1}}^{-1} D_{t_{2}}^{-1} u \in \mathcal{G}^{a_{1}, a_{2}}(\delta, L, \omega)$ et

$$
\left\|a(x) D^{\beta} D_{t_{1}}^{-1} D_{t_{2}}^{-1} u\right\| \leqslant c\left(\max \left(L^{-1}, \omega^{-1}\right)\right)^{2}\|u\| \leqslant c \max \left(L^{-1}, \omega^{-1}\right)\|u\|,
$$

vu que $L, \omega \geqslant 1$.

Q.E.D.

LEMME 3.8. - Soient $a_{1}, a_{2}>-1, \omega \geqslant 1,0<\delta \leqslant 1$ et $L \geqslant 1$ alors les opérateurs $D_{t_{i}}^{-1}, D_{t_{1}}^{-1} D_{t_{2}}^{-1}, D_{j} D_{t_{1}}^{-1} D_{t_{2}}^{-1}$ induisent des endomorphismes de l'espace $\mathcal{G}^{a_{1}, a_{2}}(\delta, L, \omega)$ de norme plus petite que $c \delta$.

Preuve. - Soit $u \in \mathcal{G}^{a_{1}, a_{2}}(\delta, L, \omega)$, on a $D_{t_{1}}^{p_{1}} D_{t_{2}}^{p_{2}} D_{t_{1}}^{-1} u=D_{t_{1}}^{p_{1}-1} D_{t_{2}}^{p_{2}} u$.

Si $p_{1} \geqslant 1$, on a

$$
\begin{aligned}
D_{t_{1}}^{p_{1}-1} D_{t_{2}}^{p_{2}} u & \\
& \ll\|u\| L^{p_{1}+p_{2}-1}\left|t_{1}\right|^{a_{1}-p_{1}+1}\left|t_{2}\right|^{a_{2}-p_{2}} D^{p_{1}+p_{2}-1} \varphi_{R}\left(\omega\left(\left|t_{1}\right|+\left|t_{2}\right|\right)+\xi\right)
\end{aligned}
$$

vu (3.3), il existe une constante $c \geqslant 0$ telle que

$$
\begin{aligned}
& D_{t_{1}}^{p_{1}-1} D_{t_{2}}^{p_{2}} u \\
& \quad \ll c\left|t_{1}\right|\|u\| L^{p_{1}+p_{2}-1}\left|t_{1}\right|^{a_{1}-p_{1}}\left|t_{2}\right|^{a_{2}-p_{2}} D^{p_{1}+p_{2}} \varphi_{R}\left(\omega\left(\left|t_{1}\right|+\left|t_{2}\right|\right)+\xi\right)
\end{aligned}
$$

d'où

$$
\begin{aligned}
D_{t_{1}}^{p_{1}} D_{t_{2}}^{p_{2}} D_{t_{1}}^{-1} u & \ll c \delta\|u\| L^{p_{1}+p_{2}}\left|t_{1}\right|^{a_{1}-p_{1}}\left|t_{2}\right|^{a_{2}-p_{2}} D^{p_{1}+p_{2}} \varphi_{R}\left(\omega\left(\left|t_{1}\right|+\left|t_{2}\right|\right)+\xi\right) .
\end{aligned}
$$

Si $p_{1}=0$, on a

$$
\begin{aligned}
& D_{t_{1}}^{-1} D_{t_{2}}^{p_{2}} u \ll\|u\| L^{p_{2}}\left|t_{2}\right|^{a_{2}-p_{2}} \sum_{j=0}^{\infty} \frac{\omega^{j}\left|t_{1}\right|^{a_{1}+j+1}}{j !\left(a_{1}+j+1\right)} D^{p_{2}+j} \varphi_{R}\left(\omega\left|t_{2}\right|+\xi\right) \\
& \ll\|u\| L^{p_{2}}\left|t_{1}\right|^{a_{1}+1}\left|t_{2}\right|^{a_{2}-p_{2}} \sum_{j=0}^{\infty} \frac{\omega^{j}\left|t_{1}\right|^{j}}{j !} D^{p_{2}+j} \varphi_{R}\left(\omega\left|t_{2}\right|+\xi\right) \\
& \ll \delta\|u\| L^{p_{2}}\left|t_{1}\right|^{a_{1}}\left|t_{2}\right|^{a_{2}-p_{2}} D^{p_{2}} \varphi_{R}\left(\omega\left(\left|t_{1}\right|+\left|t_{2}\right|\right)+\xi\right) . \\
&-642-
\end{aligned}
$$


Ceci prouve que $D_{t_{1}}^{-1} u$ appartient à l'espace $\mathcal{G}^{a_{1}, a_{2}}(\delta, L, \omega)$ et que sa norme est $\leqslant c \delta\|u\|$. On a évidemment le même résultat pour l'opérateur $D_{t_{2}}^{-1}$. Il en résulte que $D_{t_{1}}^{-1} D_{t_{2}}^{-1}$ induit un endomorphisme de $\mathcal{G}^{a_{1}, a_{2}}(\delta, L, \omega)$ de norme $\leqslant c \delta^{2} \leqslant c \delta$. De la proposition 3.7, on déduit que $D_{t_{1}}^{-1} D_{j}$ est un endomorphisme de $\mathcal{G}^{a_{1}, a_{2}}(\delta, L, \omega)$ de norme $\leqslant c \max \left(\omega^{-1}, L^{-1}\right) \leqslant c$, vu $\omega, L \geqslant 1$, donc $D_{t_{1}}^{-1} D_{t_{2}}^{-1} D_{j}$ est un endomorphisme de $\mathcal{G}^{a_{1}, a_{2}}(\delta, L, \omega)$ de norme $\leqslant c \delta$.

Q.E.D.

LEMME 3.9. - Soient $a_{1}, a_{2}>-1, \omega \geqslant 1, L \geqslant 1$ et $0<\delta \leqslant 1$ alors les opérateurs $D_{t_{1}} D_{t_{2}}^{-1}$ et $D_{t_{2}} D_{t_{1}}^{-1}$ induisent des opérateurs linéaires et continus de l'espace $\mathcal{G}^{a_{1}, a_{2}}(\delta, L, \omega)$ dans $\mathcal{G}^{a_{1}-1, a_{2}-1}(\delta, L, \omega)$ dont la norme est $\leqslant c L$.

Preuve. - Soit $u \in \mathcal{G}^{a_{1}, a_{2}}(\delta, L, \omega)$, on a $D_{t_{1}}^{p_{1}} D_{t_{2}}^{p_{2}} D_{t_{2}} D_{t_{1}}^{-1} u=D_{t_{1}}^{p_{1}-1}$ $D_{t_{2}}^{p_{2}+1} u$.

Si $p_{1} \geqslant 1$, on a

$$
\begin{aligned}
D_{t_{1}}^{p_{1}-1} D_{t_{2}}^{p_{2}+1} u \\
\left.\quad \ll\left|u \| L^{p_{1}+p_{2}}\right| t_{1}\right|^{a_{1}-p_{1}+1}\left|t_{2}\right|^{a_{2}-p_{2}-1} D^{p_{1}+p_{2}} \varphi_{R}\left(\omega\left(\left|t_{1}\right|+\left|t_{2}\right|\right)+\xi\right) \\
\quad \ll\left|t_{1}\right|^{2}\|u\| L^{p_{1}+p_{2}}\left|t_{1}\right|^{a_{1}-1-p_{1}}\left|t_{2}\right|^{a_{2}-1-p_{2}} D^{p_{1}+p_{2}} \varphi_{R}\left(\omega\left(\left|t_{1}\right|+\left|t_{2}\right|\right)+\xi\right) \\
\quad \ll L\|u\| L^{p_{1}+p_{2}}\left|t_{1}\right|^{a_{1}-1-p_{1}}\left|t_{2}\right|^{a_{2}-1-p_{2}} D^{p_{1}+p_{2}} \varphi_{R}\left(\omega\left(\left|t_{1}\right|+\left|t_{2}\right|\right)+\xi\right)
\end{aligned}
$$

vu que $L \geqslant 1$ et $\left|t_{1}\right|<\delta \leqslant 1$.

Si $p_{1}=0$, on a

$$
\begin{aligned}
& D_{t_{1}}^{-1} D_{t_{2}}^{p_{2}+1} u \\
& \quad \ll c\|u\| L^{p_{2}+1}\left|t_{2}\right|^{a_{2}-1-p_{2}} \sum_{j=0}^{\infty} \frac{\omega^{j}\left|t_{1}\right|^{a_{1}+j+1}}{(j+1) !} D^{p_{2}+1+j} \varphi_{R}\left(\omega\left|t_{2}\right|+\xi\right) \\
& \quad \ll c\|u\| L^{p_{2}+1}\left|t_{1}\right|^{a_{1}}\left|t_{2}\right|^{a_{2}-1-p_{2}} D^{p_{2}} \varphi_{R}\left(\omega\left(\left|t_{1}\right|+\left|t_{2}\right|\right)+\xi\right) \\
& \quad \ll c L\|u\| L^{p_{2}}\left|t_{1}\right|^{a_{1}-1}\left|t_{2}\right|^{a_{2}-1-p_{2}} D^{p_{2}} \varphi_{R}\left(\omega\left(\left|t_{1}\right|+\left|t_{2}\right|\right)+\xi\right) .
\end{aligned}
$$

De même pour l'opérateur $D_{t_{1}} D_{t_{2}}^{-1}$.

Q.E.D.

\section{Preuve de la proposition 2.1}

D'après (1.2), on peut écrire

$$
\mathcal{F}_{1}(u)-\mathcal{F}_{1}\left(u^{\prime}\right)=\sum_{j} h_{1, j}\left(x, z_{1}, z_{1}^{\prime}\right)\left(z_{1, j}-z_{1, j}^{\prime}\right)
$$




\section{A. Nabaji}

et d'après (2.8), on peut écrire

$$
\begin{aligned}
& \mathcal{F}_{2}(u)-\mathcal{F}_{2}\left(u^{\prime}\right)=\sum_{\beta, \kappa} h_{2, \beta, \kappa}\left(x, z_{1}, z_{1}^{\prime}\right)\left(z_{2, \kappa}-z_{2, \kappa}^{\prime}\right) z_{1}^{\beta}+ \\
& +\sum_{\beta, \kappa} k_{1, \beta, \kappa}\left(x, z_{1}, z_{1}^{\prime}\right)\left(z_{1}^{\beta}-z_{1}^{\prime \beta}\right) z_{2, \kappa}^{\prime}+\sum_{\beta, \kappa, j} k_{2, \beta, \kappa, j}\left(x, z_{1}, z_{1}^{\prime}\right)\left(z_{1, j}-z_{1, j}^{\prime}\right) z_{1}^{\prime \beta} z_{2, \kappa}^{\prime}
\end{aligned}
$$

où les fonctions $h_{1, j}, h_{2, \beta, \kappa}, k_{1, \beta, \kappa}$ et $k_{2, \beta, \kappa, j}$ sont holomorphes au voisinage de l'origine de $\mathbb{C}^{n+1} \times \mathbb{C}^{n+4} \times \mathbb{C}^{n+4}$. On peut supposer que ces fonctions sont holomorphes et bornées sur le polydisque de rayon $\eta R$ de l'espace $\mathbb{C}^{n+1} \times \mathbb{C}^{n+4} \times \mathbb{C}^{n+4}$. On pose

$$
\mathcal{V}: u \mapsto \mathcal{A}(u)+\mathcal{F}_{1}(u)+\mathcal{F}_{2}(u)+v
$$

Le problème (2.9) s'écrit alors

$$
u=\mathcal{V}(u)
$$

et il s'agit donc de démontrer que l'application $\mathcal{V}$ admet un point fixe.

On a alors la

Proposition 4.1. - Soient $a_{1}, a_{2} \geqslant 1 / q$ et $r>0$, il existe $0<\delta_{0} \leqslant 1$ tel que, pour tout $0<\delta \leqslant \delta_{0}$ et toutes fonctions $u, u^{\prime} \in \mathcal{G}^{a_{1}, a_{2}}(\delta, L, \omega)$, telles que $\|u\|_{\mathcal{G}^{a_{1}, a_{2}}},\left\|u^{\prime}\right\|_{\mathcal{G}^{a_{1}, a_{2}}} \leqslant r$, les fonctions $\mathcal{F}_{i}(u)$ et $\mathcal{F}_{i}\left(u^{\prime}\right), i=1,2$, sont bien définies, appartiennent à l'espace $\mathcal{G}^{a_{1}, a_{2}}(\delta, L, \omega)$ et

$$
\left\|\mathcal{F}_{i}(u)-\mathcal{F}_{i}\left(u^{\prime}\right)\right\|_{\mathcal{G}^{a_{1}, a_{2}}} \leqslant c L \delta\left\|u-u^{\prime}\right\|_{\mathcal{G}^{a_{1}, a_{2}}}, \quad i=1,2 .
$$

La proposition 2.1 s'en déduit aisément de la façon suivante :

On choisit $L, \omega \geqslant 1$ suffisamment grand pour que $v \in \mathcal{G}^{a_{1}, a_{2}}(\delta, L, \omega)$ et la norme de l'endomorphisme $\mathcal{A}$ soit $\leqslant 1 / 6$, puis on choisit $0<\delta_{1} \leqslant \delta_{0}$ tel que $2 \omega \delta_{1}<R$ et $c L \delta_{1} \leqslant 1 / 6$.

Soit $r \geqslant 2\|v\|_{\mathcal{G}^{a_{1}, a_{2}}}$, alors si $u$ appartient à la boule fermée $B^{\prime}(0 ; r)$ de centre 0 et de rayon $r$ de l'espace $\mathcal{G}^{a_{1}, a_{2}}\left(\delta_{1}, L, \omega\right)$, on a

$$
\begin{aligned}
& \|\mathcal{V}(u)\|_{\mathcal{G}^{a_{1}, a_{2}}} \\
& \quad \leqslant\|\mathcal{A}(u)\|_{\mathcal{G}^{a_{1}, a_{2}}}+\left\|\mathcal{F}_{1}(u)\right\|_{\mathcal{G}^{a_{1}, a_{2}}}+\left\|\mathcal{F}_{2}(u)\right\|_{\mathcal{G}^{a_{1}, a_{2}}}+\|v\|_{\mathcal{G}^{a_{1}, a_{2}}} \leqslant \frac{3 r}{6}+\frac{r}{2}
\end{aligned}
$$

d'où $\|\mathcal{V}(u)\|_{\mathcal{G}^{a_{1}, a_{2}}} \leqslant r$; ceci prouve que $\mathcal{V}\left(B^{\prime}(0 ; r)\right) \subset B^{\prime}(0 ; r)$ et, d'après (4.3) et le lemme 3.7,

$\left\|\mathcal{V}(u)-\mathcal{V}\left(u^{\prime}\right)\right\|_{\mathcal{G}^{a_{1}, a_{2}}} \leqslant \frac{1}{6}\left\|u-u^{\prime}\right\|_{\mathcal{G}^{a_{1}, a_{2}}}+\frac{1}{6}\left\|u-u^{\prime}\right\|_{\mathcal{G}^{a_{1}, a_{2}}}+\frac{1}{6}\left\|u-u^{\prime}\right\|_{\mathcal{G}^{a_{1}, a_{2}}}$ 
ceci prouve que $\mathcal{V}: B^{\prime}(0 ; r) \rightarrow B^{\prime}(0 ; r)$ est une contraction stricte, la proposition 2.1 résulte donc du théorème du point fixe.

Preuve de la proposition 4.1. - Soient $u, u^{\prime} \in \mathcal{G}^{a_{1}, a_{2}}(\delta, L, \omega)$ où $0<$ $\delta \leqslant \delta_{0}$ tels que $\|u\|_{\mathcal{G}^{a_{1}}, a_{2}} \leqslant r$ et $\left\|u^{\prime}\right\|_{\mathcal{G}^{a_{1}, a_{2}}} \leqslant r$, le lemme 3.8 montre que $z_{1, j}, z_{1, j}^{\prime} \in \mathcal{G}^{a_{1}, a_{2}}(\delta, L, \omega)$ et $\left\|z_{1, j}\right\|_{\mathcal{G}^{a_{1}, a_{2}}} \leqslant c \delta\|u\|_{\mathcal{G}^{a_{1}, a_{2}}} \leqslant c \delta_{0} r$ et $\left\|z_{1, j}^{\prime}\right\|_{\mathcal{G}^{a_{1}, a_{2}}} \leqslant c \delta\left\|u^{\prime}\right\|_{\mathcal{G}^{a_{1}, a_{2}}} \leqslant c \delta_{0} r$.

On choisit $\delta_{0}$ tel que $0<\delta_{0} \leqslant 1$ et

$$
\frac{c c_{0} \delta_{0} r}{R} \leqslant 1 / 2 \text {. }
$$

Si $F$ est l'une des fonctions $h_{1, j}, h_{2, \beta, \kappa}, k_{1, \beta, \kappa}$ et $k_{2, \beta, \kappa, j}$, le lemme 3.6 montre que $F\left(x, z_{1}, z_{1}^{\prime}\right) \in \mathcal{G}^{0,0}(\delta, L, \omega)$ et qu'il existe une constante $c \geqslant 0$ indépendante de $\delta_{0}$ telle que

$$
\left\|F\left(x, z_{1}, z_{1}^{\prime}\right)\right\|_{\mathcal{G}^{0,0}} \leqslant c
$$

ceci montre que les fonctions $\mathcal{F}_{i}(u)$ et $\mathcal{F}_{i}\left(u^{\prime}\right)$ sont bien définies.

D'une part, le lemme 3.8 montre que

$$
\left\|z_{1, j}-z_{1, j}^{\prime}\right\|_{\mathcal{G}^{a_{1}, a_{2}}} \leqslant c \delta\left\|u-u^{\prime}\right\|_{\mathcal{G}^{a_{1}, a_{2}}}
$$

et la proposition 3.5 prouve, vu les inégalités (4.5) et (4.6), que $h_{1, j}\left(x, z_{1}, z_{1}^{\prime}\right)$ $\left(z_{1, j}-z_{1, j}^{\prime}\right) \in \mathcal{G}^{a_{1}, a_{2}}(\delta, L, \omega)$ et que

$$
\left\|h_{1, j}\left(x, z_{1}, z_{1}^{\prime}\right)\left(z_{1, j}-z_{1, j}^{\prime}\right)\right\|_{\mathcal{G}^{a_{1}, a_{2}}} \leqslant c \delta\left\|u-u^{\prime}\right\|_{\mathcal{G}^{a_{1}, a_{2}}},
$$

d'où $\mathcal{F}_{1}(u)-\mathcal{F}_{1}\left(u^{\prime}\right)$ appartient à l'espace $\mathcal{G}^{a_{1}, a_{2}}(\delta, L, \omega)$ et

$$
\left\|\mathcal{F}_{1}(u)-\mathcal{F}_{1}\left(u^{\prime}\right)\right\|_{\mathcal{G}^{a_{1}, a_{2}}} \leqslant c \delta\left\|u-u^{\prime}\right\|_{\mathcal{G}^{a_{1}, a_{2}}} .
$$

Or d'après (1.2), on a $\mathcal{F}_{1}(u)=0$ si $u=0$ et donc en prenant $u=0$ ou $u^{\prime}=0$, ceci prouve, vu l'inégalité précédente, que les fonctions $\mathcal{F}_{1}(u), \mathcal{F}_{1}\left(u^{\prime}\right)$ appartiennent à l'espace $\mathcal{G}^{a_{1}, a_{2}}(\delta, L, \omega)$.

D'autre part, d'après la proposition 3.5 et l'inégalité $(4.4) z_{1}^{\beta}$ et $z_{1}^{\prime \beta}$ appartiennent à l'espace $\mathcal{G}^{q a_{1}, q a_{2}}(\delta, L, \omega)$, vu que $|\beta|=q$, et

$$
\left\|z_{1}^{\beta}\right\|_{\mathcal{G}^{q a_{1}, q a_{2}}} \leqslant c \delta^{q}\|u\|_{\mathcal{G}^{a_{1}, a_{2}}}^{q} \leqslant c \delta \text { et }\left\|z_{1}^{\prime \beta}\right\|_{\mathcal{G}^{q a_{1}, a_{2}}} \leqslant c \delta^{q}\left\|u^{\prime}\right\|_{\mathcal{G}^{a_{1}, a_{2}}}^{q} \leqslant c \delta .
$$

On a la formule

$$
z_{1}^{\beta}-z_{1}^{\prime \beta}=\sum_{j}\left(z_{1, j}-z_{1, j}^{\prime}\right) P_{j}\left(z_{1}, z_{1}^{\prime}\right)
$$




\section{A. Nabaji}

où $P_{j}$ est un polynôme homogène de degré $q-1$. On obtient, vu la proposition 3.5 et (4.4), que

$$
\left\|z_{1}^{\beta}-z_{1}^{\prime \beta}\right\|_{\mathcal{G}^{q a_{1}, q a_{2}}} \leqslant c \delta\left\|u-u^{\prime}\right\|_{\mathcal{G}^{a_{1}, a_{2}}} .
$$

La remarque 3.2 montre que si $z_{2, \kappa}$ est la fonction $u$ alors $z_{2, \kappa} \in \mathcal{G}^{a_{1-1, a_{2}-1}}(\delta, L, \omega)$ et

$$
\left\|z_{2, \kappa}\right\|_{\mathcal{G}^{a_{1}-1, a_{2}-1}} \leqslant c\|u\|_{\mathcal{G}^{a_{1}-1, a_{2}-1}} .
$$

La proposition 3.7 montre que si $z_{2, \kappa}$ est l'une des fonctions $D_{j} D_{t_{i}}^{-1} u$, $D_{j} D_{i} D_{t_{1}}^{-1} D_{t_{2}}^{-1} u$ alors $z_{2, \kappa}$ appartient à l'espace $\mathcal{G}^{a_{1}, a_{2}}(\delta, L, \omega)$, donc à l'espace $\mathcal{G}^{a_{1}-1, a_{2}-1}(\delta, L, \omega)$ et

$$
\left\|z_{2, \kappa}\right\|_{\mathcal{G}^{a_{1}-1, a_{2}-1}} \leqslant c\|u\|_{\mathcal{G}^{a_{1}, a_{2}}} .
$$

Le lemme 3.9 montre que si $z_{2, \kappa}$ est l'une des fonctions $D_{t_{1}}^{-1} D_{t_{2}}^{-1} u$, $D_{t_{2}} D_{t_{1}}^{-1} u$, alors $z_{2, \kappa} \in \mathcal{G}^{a_{1}-1, a_{2}-1}(\delta, L, \omega)$ et

$$
\left\|z_{2, \kappa}\right\|_{\mathcal{G}^{a_{1}-1, a_{2}-1}} \leqslant c L\|u\|_{\mathcal{G}^{a_{1}, a_{2}}} .
$$

De même, $z_{2, \kappa}^{\prime} \in \mathcal{G}^{a_{1}-1, a_{2}-1}(\delta, L, \omega)$ et

$$
\begin{gathered}
\left\|z_{2, \kappa}^{\prime}\right\|_{\mathcal{G}^{a_{1}-1, a_{2}-1}} \leqslant c L\left\|u^{\prime}\right\|_{\mathcal{G}^{a_{1}, a_{2}}} \\
\left\|z_{2, \kappa}-z_{2, \kappa}^{\prime}\right\|_{\mathcal{G}^{a_{1}-1, a_{2}-1}} \leqslant c L\left\|u-u^{\prime}\right\|_{\mathcal{G}^{a_{1}, a_{2}}} .
\end{gathered}
$$

Les inégalités (4.7)-(4.13) prouvent, vu la proposition 3.5, que

$$
h_{2, \beta, \kappa}\left(x, z_{1}, z_{1}^{\prime}\right)\left(z_{2, \kappa}-z_{2, \kappa}^{\prime}\right) z_{1}^{\beta} \text { et } k_{1, \beta, \kappa}\left(x, z_{1}, z_{1}^{\prime}\right)\left(z_{1}^{\beta}-z_{1}^{\prime \beta}\right) z_{2, \kappa}^{\prime}
$$

appartiennent à $\mathcal{G}^{(q+1) a_{1}-1,(q+1) a_{2}-1}(\delta, L, \omega)$, donc à l'espace $\mathcal{G}^{a_{1}, a_{2}}(\delta, L, \omega)$ puisque $(q+1) a_{i}-1 \geqslant a_{i}$ et que

$$
\begin{gathered}
\left\|h_{2, \beta, \kappa}\left(x, z_{1}, z_{1}^{\prime}\right)\left(z_{2, \kappa}-z_{2, \kappa}^{\prime}\right) z_{1}^{\beta}\right\|_{\mathcal{G}^{a_{1}, a_{2}}} \leqslant c L \delta\left\|u-u^{\prime}\right\|_{\mathcal{G}^{a_{1}, a_{2}}}, \\
\left\|k_{1, \beta, \kappa}\left(x, z_{1}, z_{1}^{\prime}\right)\left(z_{1}^{\beta}-z_{1}^{\prime \beta}\right) z_{2, \kappa}^{\prime}\right\|_{\mathcal{G}^{a_{1}, a_{2}}} \leqslant c L \delta\left\|u-u^{\prime}\right\|_{\mathcal{G}^{a_{1}, a_{2}} .}
\end{gathered}
$$

De même

$$
\begin{aligned}
k_{2, \beta, \kappa, j}\left(x, z_{1}, z_{1}^{\prime}\right)\left(z_{1, j}-z_{1, j}^{\prime}\right) & z_{2, \kappa}^{\prime} z_{1}^{\prime \beta} \\
\in \mathcal{G}^{(q+2) a_{1}-1,(q+1) a_{2}-1}(\delta, L, \omega) \subset \mathcal{G}^{a_{1}, a_{2}}(\delta, L, \omega) & \\
& -646-
\end{aligned}
$$


puisque $(q+2) a_{i}-1 \geqslant a_{i}$ et que

$$
\left\|k_{2, \beta, \kappa, j}\left(x, z_{1}, z_{1}^{\prime}\right)\left(z_{1, j}-z_{1, j}^{\prime}\right) z_{2, \kappa}^{\prime} z_{1}^{\prime \beta}\right\|_{\mathcal{G}^{a_{1}, a_{2}}} \leqslant c L \delta\left\|u-u^{\prime}\right\|_{\mathcal{G}^{a_{1}, a_{2}}} .
$$

D'où $\mathcal{F}_{2}(u)-\mathcal{F}_{2}\left(u^{\prime}\right)$ appartient à l'espace $\mathcal{G}^{a_{1}, a_{2}}(\delta, L, \omega)$ et

$$
\left\|\mathcal{F}_{2}(u)-\mathcal{F}_{2}\left(u^{\prime}\right)\right\|_{\mathcal{G}^{a_{1}, a_{2}}} \leqslant c L \delta\left\|u-u^{\prime}\right\|_{\mathcal{G}^{a_{1}, a_{2}}} .
$$

En prenant $u=0$ ou $u^{\prime}=0$, ceci prouve que les fonctions $\mathcal{F}_{2}(u), \mathcal{F}_{2}\left(u^{\prime}\right)$ appartiennent à l'espace $\mathcal{G}^{a_{1}, a_{2}}(\delta, L, \omega)$.

Q.E.D.

\section{Bibliographie}

[1] BJORK (J.). - Rings of Differential Operators, North-Holland Publishing Company Amsterdam, 1979.

[2] Deligne (P.). - Equations différentielles à points singuliers réguliers, Springer Lecture Notes, vol. 163, Berlin, 1970.

[3] Hamada (Y.), Leray (J.) et Wagschal (C.). - Systèmes d'équations aux dérivées partielles à caractéristiques multiples : problème de Cauchy ramifié; hyperbolicité partielle, J. Math. pures et appl., 55, 1976, p. 297-352.

[4] IsHII (T.). - On Propagation of Regular Singularities for Solutions of Nonlinear Partial Differential Equations, J. Fac. Sci. Univ. Tokyo, 37, 1990, p. 377-424.

[5] Kobayashi (T). - Propagation of Singularities for a First Order Semi-Linear System in $\mathbb{C}^{n+1}$, Ann. Sc. Norm. Sup. Pisa, 4, 1985, p. 173-189.

[6] LAX (P.D.). - Non Linear Hyperboliques Equations, Comm. Pure Appl. Math., vol. 6,1953 , p. 231-258.

[7] Leichtnam (E.). - Construction de solutions singulières pour des équations aux dérivées partielles non linéaires, Ann. scien. Éc. Norm.Sup., 20, 1987, p.137-170.

[8] Leichtnam (E.). - Le problème de Cauchy ramifié, Ann. scien. Éc. Norm. Sup., 23, 1990 , p. 367-443.

[9] Leichtnam (E.). - Le problème de Cauchy ramifié semi-linéaire d'ordre deux, Ann. scien. Éc. Norm. Sup., 24, 1991, p. 189-214.

[10] Nabaji (A.) et Wagschal (C.). - Problème de Cauchy ramifié pour des opérateurs semi-linéaires du second ordre à caractéristiques simples, C. R. Acad. Sci. Paris, t. 314, p. 523-526, 1992.

[11] NABAJI (A.) et WAGSChaL (C.). - Singularités à croissance lente, J. Math. pures et appl., vol. 72, 1993, p. 335-375.

[12] NABAJI (A.). - Construction de solutions singulières pour un opérateurs quasilinéaires, C. R. Acad. Sci. Paris, t. 317, Série I p. 177-180, 1993.

[13] NABAJI (A.). - Construction de solutions singulières pour un opérateurs quasilinéaires, Bull. Sci. math. 1995, 119, p. 509-527. 


\section{A. Nabaji}

[14] PongÉRARd (P.) et Wagschal (C.). - Ramification non abélienne, J. Math. pures et appl., 77, 1998, p. 51-88.

[15] Wagschal (C.). - Problème de Cauchy analytique à données méromorphes, J. Math. pures et appl., 51, 1972, p. 375-397.

[16] Wagschal (C.). - Une généralisation du problème de Goursat pour des systèmes d'équations intégro-différentielles holomorphes ou partiellement holomorphes, J. Math. pures et appl., 53, 1974, p. 99-132.

[17] Wagschal (C.). - Sur le problème de Cauchy ramifié, J. Math. pures et appl., 53, 1974, p. 147-164.

[18] Wagschal (C.). - Le problème de Goursat non linéaire, J. Math. pures et appl., 58, 1979, p. 309-337.

[19] Wagschal (C.). - Problème de Cauchy ramifié à caractéristiques multiples holomorphes de multiplicité variable, J. Math. pures et appl., 62, 1983, p. 99-127. 